\title{
L'éternel retour du mythe de Phèdre.
}

\section{Constantina SPILIOTOPOULOU}

Université Paris-IV, Sorbonne.

"Plus que de changer le monde ou que de provoquer une prise de conscience, il s'agit de

porter témoignage à long terme, de constituer un point d'appui pour quiconque, à son heure,

voudrait s'en saisir en fonction de son expérience particulière. » Yannis Ritsos

Le phénomène mythique et le phénomène littéraire existent ensemble : à l'intersection des ensembles qu'il se joue, le jeu de la répétition et de la variation, du même et de l'autre ${ }^{1}$. D'une part, le mythe s'est vu reconnaître une identité et une légitimité incontestables comme mode symbolique d'appréhension de l'expérience humaine; d'autre part, il n'est plus réservé au seul mode de pensée archaïque, à la civilisation traditionnelle, mais son polymorphisme lui permet de structurer et d'orienter représentations et actions même dans les sociétés à représentations et normes rationnelles. Cela dit, le mythe invite à une pluralité de lectures, selon les circonstances de sa réactualisation.

Dans cette optique, en étudiant les modes de présence littéraires surtout dramatiques nous visons à une meilleure compréhension au cours des siècles des enjeux de la reprise sur Phèdre par divers créateurs ainsi que des composantes ou des implications de ce mythe qui intègre toujours dans les ambiguïtés de sa composition, une part de mystère, d'effroi.

\footnotetext{
${ }^{1}$ Dans la préface du Dictionnaire des mythes littéraires qu'il a dirigé Pierre Brunel affirme que « le mythe nous parvient tout enrobé de littérature ».
} 
Au sein de la mythologie grecque, Phèdre jouit d'une place à part car elle consiste en une figure à venir dans des textes nouveaux tandis que d'autres figures comme par exemple celle de Prométhée semblent plus ou moins périmées ces dernières décennies. L'histoire de la marâtre, incapable de maîtriser son amour pour le fils de son mari, a inspiré une pléthore d' œuvres littéraires et artistiques de sorte que son personnage devenu légendaire a pu passer dans la sphère du mythe. Au moment de l'avènement de la condition postmoderne, des flots d'encre continuent à couler sur la figure de Phèdre, sur le conflit de la Conscience et de la Passion, du Caractère et du Destin. En effet, Phèdre témoigne de conflits inextricables entre passions et intérêts politiques, corps et Etat. S'y enchevêtrent avec une violence rare les grands sujets politiques - territoire, guerre, filiation - et obscènes -adultère, inceste. Conflits d'identités, de pouvoir, de modèles sociaux et civils : autant d'aspects qui nous poussent à réfléchir sur la «modernité » antique de Phèdre, comme femme, coupable ou victime, puissance ancienne et terrible, maillon faible de guerres dans lesquelles est en jeu le futur générationnel de l'humanité. Un texte et des thèmes souvent relus et re-contextualisés ${ }^{2}$ par différents auteurs. Euripide, Sénèque et Racine restent toujours les principaux modèles dont les dramaturges s'inspirent pour mettre en scène la passion néfaste de Phèdre qui devient plus ou moins violente dans son développement.

Avec cette contribution, nous souhaiterions réfléchir une fois encore sur le mythe de Phèdre dans une approche à la fois synchronique et diachronique. Nous tenterons donc de concilier deux objectifs: effectuer une présentation des principales lectures/réécritures de cette figure mythique et proposer quelques pistes de réflexions à partir de l'étude du cas de Phèdre. Quoi

2 Le concept de "recontextualisation" permet de rendre compte de ce mécanisme par lequel la littérature utilise les traces mythiques. 
qu'il en soit, nous accorderons la plus grande importance à ses versions théâtrales parmi les nombreuses interprétations littéraires, cinématographiques ou artistiques de son mythe de l'Antiquité jusqu'aux œuvres contemporaines. Pourquoi , entraîne-t-il autant de réécritures ? De quelle façon convoque-t-il enjeux et conflits toujours actuels ? Tout d'abord, nous allons donc considérer ce mythe, sa fortune, ses caractères dans le théâtre antique à Athènes et à Rome et après à Paris au XVIIe sicle. Ensuite, sa réception contemporaine nous amènera à nous intéresser aux différentes transformations qu'il a subies dans les cultures d'arrivée. A voir, à travers des exemples contemporains en juxtaposition avec les grands auteurs classiques, comment il a été repris, modifié, adapté pour devenir un mythe moderne de sorte qu'on peut parler d'une Phèdre moderne.

Le sujet oblige à retourner aux sources littéraires antiques. Le nom de Phèdre le fait remonter à des temps mythiques. La plus ancienne mention de Phèdre est homérique ${ }^{3}$, celle d'Hippolyte $^{4}$ se trouve aussi dans la poésie épique, mais dans aucun de ces deux textes il n'est établi de rapport entre les deux personnages. On le voit, l'étude des grands mythes littéraires s'appuie sur quelques œuvres que' l'on s'accorde à considérer comme grandes, voire immortelles. Il faut au moins une tragédie, et l'on apprécie qu'elle soit signée d'un grand nom. A quelques détails près, les données de base changent peu dans l'Antiquité, et l'action est d'une simplicité linéaire. La mère de Phèdre, Pasiphaé et sa sœur Ariane sont possédées par des désirs hors du commun.

\footnotetext{
${ }^{3}$ Homère, Od., XI, v. 321-325. Mais la mention de Dionysos dans ce passage indique qu'il s'agit d'une interpolation que l'on ne saurait faire remonter au-delà du Vle siècle.

${ }^{4}$ Les Chants de Naupacte (épopée des VIle-Vle siècles, consacrée aux femmes célèbres selon Pausanias, $X, 38,6$ et attribuée à un certain Carcinos), s'il faut en croire un passage (du reste peut-être interpolé) de la Bibliothèque du Pseudo-Apollodore (III, 10,3) et un fragment du Péri eusébéias de Philodème (fr. 11, EGF, Kinkel, p. 202), mentionnaient Hippolyte.
} 
Pasiphaé s'était amourachée d'un taureau et Ariane finit par s'unir à Dionysos. La petite fille du Soleil, Phèdre « la brillante » est maudite. Elle s'éprend de son beau-fils, Hippolyte, et, le trouvant insensible, l'accuse auprès de son époux, Thésée, d'avoir voulu la séduire, puis se donne la mort. Thésée, furieux, invoque la vengeance de Poséidon, qui envoie un monstre marin contre son fils innocent. Ce mythe de Phèdre a inspiré, du moins d'après les œuvres qui nous restent, deux grandes tragédies dans l'Antiquité, une grecque et une romaine. Il s'agit de l'Hippolyte porte-couronne d'Euripide (428 av. J.-C.) ${ }^{5}$ et de la Phèdre de Sénèque ${ }^{6}$. Si on est obligé de partir d'Euripide, c'est qu'il a été le premier, à notre connaissance, à proposer une véritable étude de caractère, non seulement de Phèdre elle-même, mais des personnages qui entrent en contact avec elle. Il semble en tout cas que la modernité d'Euripide a séduit maints créateurs de notre époque; Jules Dassin et Marguerite Yourcenar parmi d'autres ont été retenus par le réalisme du grand tragique grec. La pièce a ceci de particulier qu'elle ne n'intéressait pas au personnage de Phèdre. Le véritable personnage tragique au cœur de la pièce est Hippolyte. A partir de la première manifestation dramatique du mythe, Phèdre n'aura pas d'existence indépendante sans Hippolyte ; qui pense aujourd'hui à Phèdre songe tout de suite à Hippolyte. Ce sont les circonstances décrites par Euripide qui créent Phèdre : dès son apparition chez lui, hors d'un certain « climat » elle disparaît de la littérature.

\footnotetext{
${ }^{5}$ Euripide en a fait le sujet de deux de ses pièces. La première, Hippolyte voilé (titre qui n'est pas d'Euripide), datée peut-être de 432-430, est perdue, à l'exception d'une vingtaine de fragments (TGrF Nauck2, fr. 428-448) ; la seconde est celle de 428 qui est conservée. Entre les deux Hippolyte d'Euripide (selon l'hypothèse de W. S. Barrett, Euripides, Hippolytos, op. 12-14), Sophocle a donné, peut-être en 429, une Phèdre, dont il reste quelques fragments.

${ }^{6}$ Senecae L. Annei, Phaedra: Sénèque Phèdre: Edition, introduction et commentaire, Paris, Presses Universitaires de France, 1965.
} 
Euripide met l'accent sur la déchéance morale de son héroïne. Phèdre se réduit chez lui à un pur moyen de vengeance utilisé par Aphrodite contre Hippolyte. Elle parle de l' $\dot{\alpha} \mu \alpha \rho \tau i \alpha$ (le péché) qu'elle veut éviter, un discours qui la replace dans le contexte plus large de la bonne conduite que doivent observer les reines:

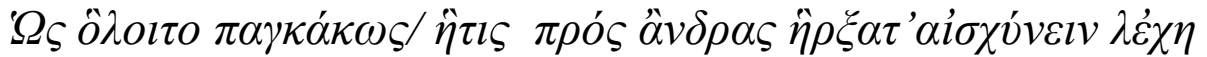

Périsse de malemort celle qui, la première, osa déshonorer sa couche ! ${ }^{7}$.

Il n'est guère question de la faute de Phèdre. Euripide présente le thème comme un conflit entre deux déesses, Aphrodite, la patronne de l'amour physique contre Artémis - la déesse de la chasse, détournant ainsi la pièce des puissances humaines, alors qu'il est le peintre par excellence du «drame de la passion érotique ${ }^{8} \gg$ qui se plaît à exposer la force d'Eros dans sa dimension psychologique en montrant ses ravages sur Phèdre. En outre, si Phèdre est l'enjeu de cette lutte, celle-ci se déroule toutefois au-dessus d'elle. Elle n'a aucune indépendance, volonté propre. Dans l'œuvre d'Euripide, Hippolyte, de son côté, «celui qui lie les chevaux» comme nous l'indique son étymologie, est poursuivi par Aphrodite. Au lieu de faire des prières à la déesse de l'amour physique, il préfère la chasteté et la pureté de la déesse chasseresse. Sa chasteté excessive est un sacrilège, une offense aux yeux d'Aphrodite. Son action déclenche ainsi le conflit tragique. Dès lors, nous allons retrouver à plusieurs reprises l'ambiguïté du personnage qui oscille entre sauvage des forêts et défenseur de la pureté morale. Chez Euripide, l'antagonisme profond qui oppose la passion à la pureté, Aphrodite à Artémis, s'extériorise dans la dramatique rencontre de Phèdre et d'Hippolyte. Aphrodite va rendre Phèdre

\footnotetext{
${ }^{7}$ Euripide, Hipp., v. 407-409.

${ }^{8}$ A. Lesky, La Tragédie grecque. Présentation, traduction et indu par M. Lebel Mediaspaul, Sherbrooke, 1997, p. 202.
} 
folle amoureuse d'Hippolyte. Il est à noter que dans la pièce d'Euripide Phèdre n'ose pas à avouer son amour à Hippolyte et ne parvient pas à prononcer son nom :

Phèdre: $\mathrm{T} u$ vas ouïr le comble des horreurs.

J'aime ... A ce nom fatal, je tremble, je frissonne.

J'aime...

Oenone (la nourrice) : Qui?

Phèdre: Tu connais ce fils de l'Amazone, Ce prince si longtemps par moi-même opprimé?

Oenone: Hippolyte! Grands Dieux!

Phèdre: C'est toi qui l'as nommé.

Alors, c'est la nourrice qui se charge de révéler les sentiments de sa maîtresse au fils de Thésée. Nous ne savons pas en quels termes la nourrice lui a fait cette annonce, puisque les deux personnages sont à l'intérieur du palais pendant que Phèdre essaie de comprendre, de l'extérieur, ce qui s'y trame (Hipp. ver. 565600). Dans le passage précédent, communément nommé par la critique " scène des aveux de Phèdre », (une scène qui va réapparaître jusqu'aujourd'hui) le problème auquel doit faire face l'héroïne est de savoir comment dire un amour interdit, un amour qui flétrit son honneur.

Désormais pour le chaste Hippolyte, fils d'une chaste Amazone, la sexualité féminine est une monstruosité. Phèdre éprise d'un amour coupable pour son beau-fils va se laisser complètement aller à son désir aveugle ${ }^{9}$. Désespérée, elle simule un viol. Thésée revenant des Enfers condamne son fils. Un messager arrive et raconte la mort d'Hippolyte, qui s'est fait surprendre, dirigeant son char, par un gigantesque taureau, envoyé par Poséidon. Le récit, magnifique, est profondément

9 D'une façon générale, les passions entraînent, dans le théâtre d'Euripide, toutes sortes de violences dues au désir de rendre coup pour coup, de faire souffrir parce qu'on souffre ", remarque Jacqueline de Romilly. 
émouvant et constituera un des plus beaux morceaux plus tard de la littérature française, sous la plume de Racine. La description est déjà formidable chez Euripide, et sans doute constitue un des éléments qui lui valurent, cette année-là, le premier prix du concours tragique. En apprenant la mort de celui qu'elle aimait, Phèdre se pend et Thésée se rend compte de son abominable erreur. L'effet de surprise est total car Euripide a choqué le public athénien en mettant de cette manière en scène le désir sexuel féminin ${ }^{10}$. Avec Phèdre, la femme cesse d'être un objet de plaisir pour devenir un sujet désirant ${ }^{11}$. Déjà dans Hippolyte porte-couronne Euripide fait de la femme une puissance infernale, une hypostase de la mort, dotée pour l'homme comme pour elle-même d'une puissance mortifère sans mesure. En ce sens, Phèdre est une figure féminine mythique représentative de cette puissance de mort. Dès Euripide jusqu' à nos temps par Phèdre et Hippolyte il apparaît que le thème de l'amour où l'adultère se mêle à l'inceste est indissociablement lié à celui de l'infanticide.

Désormais l'amour est un poison versé dans son cœur par une divinité ennemie. Dire que Phèdre a été frappée d'égarement par Aphrodite, c'est à la fois lui permettre de se considérer comme une victime et proclamer qu'elle est en contact avec le sacré. Quand on compare la tragédie Hippolyte porte-couronne à la tragédie Hélène on est frappé de voir que les deux textes

\footnotetext{
10 Selon Jacqueline de Romilly dans "Les réflexions générales d'Euripide : analyse littéraire et critique textuelle", Comptes-rendus des séances de l'Académie des Inscriptions et Belles-Lettres ", 127e année, N. 2, 1983, pp. 405-418, nous pourrions expliquer les libertés que prend Euripide à la lumière des changements dans Athènes de la fin du Ve siècle, une période pendant laquelle on a commencé à se passionner pour l'étude de la nature morale humaine.

${ }^{11}$ Sur le désir féminin dans la tragédie grecque, par nature incontrôlé et dangereux pour les hommes, voir A. Carson, Putting her in her place : woman, dirt, and desire, dans Before Sexuality. The construction of erotic experience in the ancient Greek world.
} 
d'Euripide font place au caprice de certaines déesses et à leur pouvoir mystérieux ; on voit le sacré s'insinuer dans les mortels, effacer les limites de l'individu. Dans Hippolyte porte-couronne le choix simplificateur qui amène les personnages à honorer Artémis ou Aphrodite se révèle tragique. Pour participer à la vie ils doivent donc réaliser en eux la synthèse d'influences contradictoires. Mais il faudrait tout de même ajouter que Phèdre $\mathrm{y}$ est à la fois une victime et un objet d'horreur. Il y a là un accroc inattendu dans le tissu serré de la morale classique.

De plus, il faut souligner une ambiguité du personnage de Phèdre. Chez Euripide, elle est une fausse $\gamma v v \eta ́$ parce qu'elle ne connaît pas le lit de l'accouchement qui est le même que celui du mariage. Seule la première maternité permet à une jeune mariée de s'accomplir comme $\gamma v v \eta$, mère-épouse. Il apparaît que Phèdre ne dispose pas des moyens pour pouvoir retrouver sa place dans la vie normale de la cité. Par ailleurs, Phèdre chez Euripide tout comme sa cousine Médée constitue une figure du déplacement féminin et de destruction. Elles sont des étrangères dans la cité, trahies et héroïnes des crimes passionnels. En même temps, dans La Poétique, Aristote indique que les héros de tragédie, contrairement aux personnages de comédie, sont de noble extraction. Les conflits tragiques sont donc à la fois personnels et politiques : la mort d'Hippolyte ou l'adultère de Phèdre ont des conséquences au plus haut niveau de la Cité. Il faut enfin savoir que la passion mortelle de Phèdre est une atteinte à l'ordre patriarcal dont sont ici rapprochées les deux composantes, pouvoir des hommes sur les femmes et des pères sur les fils, ordre patriarcal auquel adhère Hippolyte et qu'il a reçu mission de préserver.

Les Romains se situent quant à eux dans la filiation littéraire des Grecs. Les tragédies romaines ${ }^{12}$ s'inspirent des

\footnotetext{
${ }^{12}$ Introduit dans la littérature latine, le thème fut repris par Ovide dans les Métamorphoses (XV, vv. 487 sqq.), et dans les Fastes (III, vv. 265 sqq.) ; les premières, comme du reste le
} 
dramaturges grecs et certaines comme Phèdre de Sénèque ${ }^{13}$ ont à leur tour inspiré le théâtre classique contemporain ${ }^{14}$. Même si le souvenir d'Euripide est parfois très net, Sénèque ajoute des aspects spécifiques à la société romaine antique. La pièce de Sénèque annonce une nouvelle interprétation du mythe, dans la mesure où c'est Phèdre qui devient le personnage central du drame. Sénèque l'étoffe en même temps qu'il la responsabilise et la dote d'une véritable épaisseur psychologique. Phèdre est surtout sensible et vulnérable, mais c'est à elle seule qu'elle doit de ne pas se Iaisser engloutir dans le tourbillon de Ia passion. La preuve est faite : le combat, tragiquement, est en chacun, mais il est de notre ressort de l'emporter. D'ailleurs, pour qu'une bonne tragédie réussisse à Rome, il faut qu'elle soit horrible à souhait. Deux ingrédients en assurent le succès : sang et folie (le furor) ${ }^{15}$. Les caractéristiques de la Phèdre de Sénèque sont essentiellement romaines. Sénèque semble mettre en relief l'image d'une femme furieuse, qui agit sans raison, prise dans une sorte de folie. En effet, Sénèque fait de ses personnages mythologiques des êtres troubles. Quant au sang, l'annonce faite

\footnotetext{
septième chant de l'Enéide (vv. 761 sqq.) mentionnent la mort d'Hippolyte, sa résurrection et sa transformation en Virbius; les secondes, avec l'Énéide rapportent que les chevaux, instruments de la mort du héros, étaient exclus du bois où se vivaient après cette métamorphose.

${ }^{13}$ Sur la Phèdre de Sénèque, voir Pierre Grimal, «L'originalité de Sénèque dans la tragédie de Phèdre ", Revue des études latines, XLI, 1963, p. 297-314.

${ }^{14}$ A cet égard, les tragédies classiques sur Hippolyte et Phèdre deviennent un espace foncièrement intertextuel. Les maintes références à Euripide et à Sénèque dans le paratexte des textes écrits jusqu'aujourd'hui prouvent que leurs auteurs se réclament d'eux dans un désir de légitimation littéraire.

${ }^{15}$ Le théâtre de Sénèque est également profondément structuré par la notion de furor comme le démontrent les travaux de Florence Dupont. Tous les héros criminels de la tragédie latine agissent en proie à une

folie, le furor, qui les conduit à commettre le crime tragique, le scelus nefas, lequel, dans la tragédie latine, est toujours un crime monstrueux, horrible, un crime contre l'ordre sacré du monde.
} 
à Thésée de la mort de son fils n'épargne guère de détails... Ce texte de Sénèque est somptueux, parsemé de joyaux incandescents et poétiques, riche de mille thèmes: la nostalgie d'un ordre originel, les considérations morales et politiques, jusqu'à la description de la mort d'Hippolyte poursuivi par la malédiction paternelle. La main de Neptune n'est pas guidée par la colère des dieux, mais par la folie de l'homme. La Phèdre latine est une passionnée qui subit avec frénésie l'amour qui la dévore. Signe des temps, l'homme s'est affranchi, et au siècle de Sénèque, les dieux et démons ne sont plus dans le ciel, au-dessus de nous mais dans la société humaine. Moraliste et fin observateur des passions humaines, Sénèque n'a pas le sens du merveilleux. Par ailleurs, Sénèque ne peut faire l'impasse de la philosophie stoïcienne, de sa physique et de sa morale. Le théâtre de Sénèque devient un théâtre qui traite de la puissance de la destruction, de la folie à l'œuvre dans Rome. Plus que la Phèdre grecque, la Phèdre "romaine » se caractérise par sa violence, sa cruauté. Sur un plan strictement métaphorique, elle représenterait une Rome que l'on a souvent peinte comme étant le symbole des maux divers. La pièce de Sénèque illustre un moment particulier qui témoigne d'une modernité: dans l'histoire de la pensée d'abord, dans la mesure où sa conception de l'homme annonce le courant de la pensée moderne ; mais aussi dans l'évolution du genre tragique. En d'autres termes, le texte latin, nous semble constituer une étape importante dans le voyage du mythe de Phèdre dans la diachronie.

Les classiques français du XVIIe siècle ${ }^{16}$, comme c'est le cas de la Phèdre de Racine avec la Phèdre de Sénèque ${ }^{17}$ sont

\footnotetext{
${ }^{16}$ Quant aux nombreux ouvrages français composés sur le même thème, ils comprennent les Hippolyte de Garnier (1573), de La Pinelière (1635), de Gilbert (1646) et de Bidar (1675), tandis que de Tristan l'Hermite, nous avons un long poème sur la mort du héros (1637). La Phèdre et Hippolyte de Pradon est légèrement postérieure à la tragédie de Racine; elle fut représentée deux jours après celle-ci, le 3 janvier 1677.

${ }^{17}$ L'influence du théâtre de Sénèque est à elle seule un objet d'étude imposant; voir la
} 
dans une relation intertextuelle explicite avec les classiques gréco-latins

La réminiscence des œuvres raciniennes est un passage obligé pour quiconque fait la lecture des tragédies écrites dans le sillage de leur postérité. La huitième tragédie écrite par Racine, juste avant son silence littéraire, est considérée par son auteur même comme le chef d'œuvre de sa création. Cependant le mythe de Phèdre est chez lui, le produit de plusieurs siècles d'élaboration continue, le résultat des apports successifs de chaque génération qui a fourni son lot de poètes et d'exégètes. La Phèdre de 1677, une fille de la famille royale de Crète, par une adaptation parfaite de Racine, se transforme en une femme française. Elle est une véritable reine, qui se torture par une passion implacable, invincible, involontaire et intensive. L'infidélité amoureuse de Thésée l'a brisée et elle voit naître une passion incestueuse en elle, une passion pour son beau-fils. Parmi les riches analyses sur ce point, toutes dignes d'intérêt, nous pourrions garder celle de Lucien Goldmann ${ }^{18}$, philosophe plus qu'historien. A ses yeux, cette tragédie racinienne présente la vision tragique dans sa perfection: «l'illusion et la faute de Phèdre incarne l'homme tragique, le juste pécheur». Phèdre comprend très bien que c'est un amour interdit, elle résiste comme dans le texte d'Euripide, et elle fléchit. Mais Phèdre, quel que soit son caractère, est avant tout une femme, que l'on représente généralement comme la plus violemment tourmentée des héroïnes raciniennes. Elle possède quelque chose de fin, comme la plupart des autres. Son caractère et ses pensées sont beaucoup plus complexes que ceux d'Hippolyte chez Euripide. Sa figure symbolise une force

thèse soutenue en novembre 2004, à la Sorbonne, par Florence de Claigny : Imitation, traduction et adaptation des tragédies de Sénèque aux XVle et XVIle siècles en France.

${ }^{18}$ Golddmann Lucien, Le Dieu caché. Etude sur la vision tragique dans les Pensées de Pascal et dans le Théâtre de Racine. Paris, Gallimard, 1955. 
sociale, si on dit qu'Hippolyte représente une nature ou un état sauvage. En fait, comme le souligne Racine dans la préface de l'œuvre, elle n'est pas tout à fait innocente, ni tout à fait coupable. Il y a, chez elle, des contradictions qu'on lit souvent concernant l'humanité. L'Hippolyte d'Euripide et la Phèdre de Racine, montrent deux pôles humains ${ }^{19}$. L'un est la nature, un trait primitif, instinctif; l'autre est la socialisation, un trait civilisé. Bien sûr, ici, pensons aussi au fait que Racine a modifié Hippolyte dans sa pièce, orientant ainsi le mythe dans une direction toute nouvelle. Ce jeune homme qu'on trouve farouche et misogyne chez Euripide, devient un prince élégant, un noble à la française. Son refus d'amour pour Phèdre comprend une nouvelle cause : il sait aimer, mais il aime une autre fille, Aricie. En plus, les sentiments exprimés par tous les personnages sont souvent incomplets. Ce que Racine cherche, c'est écrire les mortels dans le monde de la mondanité de son époque. Racine, bien qu'il connaisse la littérature grecque, ne peut pas écrire ses drames à la grecque, car la religion sur laquelle les auteurs basent leurs œuvres a profondément changé. Il sert aussi de moraliste, avec tout ce que la religion et la société du XVIIe siècle lui ont apporté. Il nous raconte un conflit entre la passion et la raison, le sort de Phèdre en qui la passion a vaincu la raison, n'est que la destruction de soi-même et d'autrui. Sans oublier que Racine tourne son regard des dieux vers les hommes. Tout rentre dans l'ordre du Destin : les dieux ont terrassé les hommes, l'Eros a vaincu l'Ethos, le Mal l'a emporté sur le Bien. Le génie de Racine aura été d'opérer une synthèse personnelle entre un moule commun hérité, et une fidélité littéraire à l'esprit de la tragédie antique. Et ceci en imaginant d'étendre le champ de l'application de la lutte entre proches de condition royale au champ clos, de la conscience individuelle saisie dans sa relation

${ }^{19}$ Forestier, Georges. "Introduction ", dans Racine, CEuvres complètes, I, Théâtre - Poésie, éd. G. Forestier, Paris, Gallimard, Bibliothèque de la Pléiade,1999, p. XLI et sq. 
existentielle à soi et au monde. La réussite de sa thèse implique nécessairement que l'homme se délivre de l'ignorance et entre dans une nouvelle phase plus civilisée. Certes, on conçoit que Racine ait été porte vers des figures de la tragédie préchrétienne comme celle de Phèdre. Phèdre transposée dans un siècle que dominent encore solidement les préceptes chrétiens, elle y prend une allure exotique, un relief plus dépouillé et plus inquiétant : elle a la charge d'exprimer l'interrogation primordiale de l'être perdu de Dieu, frustré dans son amour, détourné par des forces inconnues, embrouillé par cela qui devait le sauver. C'est la monstration du mal dans sa logique rigoureuse, son triomphe sans recours, qui finira par écraser l'auteur lui-même, le réduire au silence. C'est Phèdre le dernier mot de Racine jusqu'à nous...

Bien que ces trois œuvres aient une liaison directe et remarquable, il y a de profondes diversités entre elles. Ces diversités ne sont pas un simple changement de mœurs ou de religions, mais plutôt une évolution de thème, de structure et de figures des personnages. A cela, il faut ajouter que chacun des auteurs mentionnés a écrit à un moment de rupture sociopolitique important, et ce sont ces crises qui continuent à intéresser. C'est-à-dire la manière dont la machine théâtrale d'une époque (langue, corps, scène, finances, public,...) reflète ses idéologies, ses peurs, ses combats, ses renoncements. Si telles sont les assises de la pensée mythique ancrée dans la figure de Phèdre, il s'agirait d'observer, dans le cadre de nos préoccupations, les modifications aussi bien que les différentes formes de continuité dans ce domaine - telles que conditionnant, nourrissant la pensée contemporaine.

L'histoire de Phèdre pourrait s'achever ici ; mais elle est ainsi faite qu'elle est un éternel recommencement, une continuelle montée de sève. Le critique que l'on interrogerait sur l'éventuel succès du mythe de Phèdre dans les lettres 
contemporaines, n'émettrait vraisemblablement aucun doute quant à son exceptionnelle fortune. Qui veut se pencher sur le mythe de Phèdre se trouve donc en présence d'un scénario mythique toujours vivant et assez clairement identifiable et de traditions interprétatives attestées, dont les écrivains se sont inspirés et qu'ils ont à leur tour enrichies. Sa première référence pourrait être la résurgence des mythes grecs en général, et surtout des mythes féminins : de Cocteau à Anouilh, que de Médées, d'Antigones, d'Electres, de Phèdres ! Mais, par delà de ces figures mythologiques féminines telles qu'elles arrivent jusqu'à nous (" pétries d'imaginaire », selon la belle expression de l'historien Jacques Le Goff), un champ immense continue d'être ouvert à l'exploration. Leur éviction de la polis, leur nonreconnaissance au cœur de celle-ci, trouverait son contrepoint dans leurs gestes de tisserandes de la vie humaine contemporaine.

Dans un nouvel effort, rompant avec les traditions, l'extrême fin du XXe et le début du XXIe siècle restent ouverts aux formes puissantes du trésor légendaire de la Grèce qui créent les conditions d'une nouvelle lisibilité. Au vu de ce qui précède, de l'Antiquité à nos jours, les auteurs n'ont cessé d'écrire Phèdre, de la rapprocher de nous. Le personnage de Phèdre continue à fasciner le XXIe siècle qui ne paraît pas disposé à rompre avec l'habitude de réécrire la vie, les pensées, les déchirements de la passionnée coupable ou innocente par excellence. Tous les ans, des œuvres littéraires consacrées à Phèdre et à Hippolyte s'ajoutent à une très longue liste de tous les pays du monde.

La Phèdre qui vient à l'esprit des auteurs du XXe et XXI siècles est parmi les dernières alluvions déposées par le grand fleuve de l'écriture et des lectures du mythe; elle n'a que de lointains rapports avec les strates les plus anciennes. Les écrivains contemporains en transposant le mythe de Phèdre se 
sont servis des procédés stylistiques tels que la banalisation et la dégradation pour tourner les personnages du mythe antique en dérision. L'inversion des situations mythiques leur permet de donner une nouvelle version significative du mythe antique.

De nos jours, le mythe sera inscrit dans une filiation littéraire contemporaine en rendant le passé de Phèdre présent. Dans une perception cyclique de l'Histoire - typique du mythe, dira-t-on - que met en relief Mircea Eliade, une sorte d'équilibre s'établit par le biais de la conjonction entre plusieurs phases de destruction suivies de construction. Si on observe de près le mythe de Phèdre et ses manifestations, on se rend compte que ce sont là les mouvements typiques de ce paradigme ${ }^{20}$.

Dans l'ensemble, les auteurs nouveaux aimeront mieux créer sur Phèdre que se souvenir. Il faut noter, d'ailleurs, qu'ils ne retiennent des interprétations passées que les éléments de comparaison «littéraire ». Parvenir à inventer une autre écriture sur Phèdre dans un monde qui ne conserve comme forme de représentation que des bribes du mythe antique, c'est tenter souvent, de proposer une autre esthétique de la réception. Quels sont alors ces éléments de la légende qui trouvent audience aujourd'hui et qui constituent l'origine de la conception moderne de Phèdre et d'Hippolyte?

\footnotetext{
${ }^{20}$ On peut encore citer Ihab Hassan pour qui la littérature contemporaine est placée dans le postmodernisme et fortement marquée par le déchirement. En plus, la nature éclatée des œuvres de notre temps ne dit cependant pas "tout» sur leur fonctionnement. La mythocritique nous a certainement appris à voir ce phénomènes dans sa mouvance (Durand, Figures mythiques...), dans sa pluralité: jamais un procédé ne se présente, dans une création de la pensée, sans son contraire, sans sa partie "ombre» (avec un héros pastor et agnus, rédempteur et victime), et on a appris à lire (encore chez Durand) qu'aucun «régime» n'apparaît séparé de son contraire. Dans le volume intitulé Mythocritique. Théorie et parcours Brunel essaie aussi de rassembler à ce propos quelques éléments théoriques en recherchant la présence du mythe chez quelques auteurs et dans un certain nombre de textes, à partir de la conviction que la mythocritique "s'intéressera surtout à l'analogie qui peut exister entre la structure du mythe et la structure du texte ».
} 
D'une manière générale le mot sang d'une œuvre à l'autre se réfère à la généalogie, à l'histoire individuelle. Parallèlement se dessine une vision du mot «joug » qui rappelle toujours les entraves et les contraintes imposées par les règles collectives aux individus. Il est curieux d'observer, à travers l'histoire du mythe, la lente transformation de l'idée de la violence. La violence réside en premier lieu dans la matière du mythe, dans l'histoire ancienne où l'on explore les relations cruelles qu'entretient un individu avec ces deux groupes que sont la famille et la société. Mais on pourrait être conduit à penser que ce qui fascine les auteurs contemporains n'est pas tant cette violence originelle mais la violence issue de sa répétition éternelle. À l'échelle supérieure, sans surprise, l'organisation politique et culturelle de notre monde reproduit les mêmes schémas anciens. La société est un système organisé en fonction de la satisfaction du plus puissant. Dans la littérature dramatique actuelle, la famille royale athénienne devient ainsi objet de projection fantasmagorique pour son peuple souvent terriblement brutalisé. On peut aussi remarquer que la guerre ou la crise politique sans être aucun des sujets de ces pièces modernes, sont présentes comme une trame de fond, impossible à faire disparaître, accentuant encore les images d'un monde en destruction. La littérature dramatique apparaît comme sous le coup d'une fascination-répulsion pour les traumatismes de l'histoire récente, les catastrophes. Elle utilise, par exemple, la tradition héritée de Phèdre et Hippolyte comme autant de métaphores pour dire la barbarie du monde. En plus, le déchainement de la cruauté comme la transgression des tabous confirment, s'il était nécessaire, que ce n'est pas l'admiration pour les figures lumineuses de la résistance, de la mémoire, de la dignité face à l'outrage ou de l'affirmation de la liberté face aux arrêts du destin qui motive la reprise des matériaux mythiques par les auteurs d'aujourd'hui. 
L'étude des modalités et de la portée de la transposition du mythe d'un siècle à l'autre nous révèle que la passion continue d'animer Phèdre la souffrante. La voici à nouveau attachée désespérément à l'angoisse qui lui a été imposée pour prix de sa passion. L'homme n'a pas besoin d'avoir constamment devant les yeux l'objet de sa passion ; il le recrée sans cesse la mémoire, l'imagination, le raisonnement, par la magie du verbe, toute une poésie passionnelle dont Phèdre offre le sublime exemple. «Ma passion, c'est moi, et c'est plus fort que moi » : constat fondamental d'où naît le tragique. Mais difficile de discerner dans cet aspect du tragique le bien et du mal. Les valeurs s'ébranlent, la morale se met en question. La satisfaction est impossible et l'affirmation de soi la question fondamentale. Le désir absolu, cette force invisible, monstrueuse, envahit le corps humain et se présente assoiffé. Il bouleverse tout équilibre, individuel et social. Et à ce niveau, il est possible d'apercevoir l'imposture, la plus énigmatique de toutes les figures du destin, car elle altère la personnalité de Phèdre qui garde pourtant jusque dans ce naufrage la pleine conscience et la jouissance de soi ; elle est le suicide de ce qui ne peut pas être tué. Cette irrépressible lucidité subsiste entièrement dans le paroxysme amoureux de Phèdre. Sous cet angle, dans la recherche de l'union avec l'autre, l'individualité de la femme se trouve menacée et non constituée. On pourrait même dire pour reprendre Elisabeth Badinter $^{21}$, qu' isolés l'Un [l'homme] de l'Autre [la femme], ils (dans notre cas, Hippolyte et Phèdre) semblent à la fois inutiles et en danger de mort, comme si seule l'unité des deux pourrait avoir sens et efficacité. Ainsi, seule solution la mort, autrement dit, le silence, ce silence salvateur d'où tout son peut naître à nouveau. La mort constitue l'autre élément majeur par lequel la littérature contemporaine ramène à elle la figure de Phèdre. Quitter l'enfer

${ }^{21}$ Badinter Elisabeth, L'un et l'autre, Odile Jacob Paris, 1986, p. 23. 
de notre monde, comme Phèdre et Hippolyte, est l'ultime moyen - paradoxal, sans doute - de témoigner que notre civilisation est fondée sur la mort. La mort sert la construction mythique dans la mesure où elle permet l'expression de fantasmes propres à l'imaginaire décadent : ceux d'un érotisme morbide qui se marie fort bien à l'auréole intellectuelle qui vient d'être évoquée. En somme, ces thèmes font bien partie de l'héritage ancien de Phèdre.

Certes, en dépit de la distance linguistique et historique, le personnage de Phèdre semble d'une modernité et d'une actualité saisissantes. Dans ce sens, on a pu dire que Phèdre devient $t$ une héroïne moderne qui subit des formes nouvelles du destin tragique. On note souvent que les épisodes conduisent, conformément à la tradition classique, un peu plus près de la catastrophe. En général, quelle que soit la perspective artistique, le retour au mythe de Phèdre respecte la plupart du temps les structures dramaturgiques de la tragédie grecque et préfère la réhellénisation complète ou partielle des noms propres.

Nous remarquons également qu'on se rend compte que le personnage de Phèdre se retrouve dans plusieurs cultures ou sociétés selon le contexte qui lui est particulier. Cela dit, toute société peut se prévaloir d'une Phèdre. Il suffit de regarder ce qui se passe sur les scènes du monde pour bien se persuader ce qui fait les spécificités «nationales» de cette écriture sur Phèdre. Voilà un mythe qui traverse des cultures à la fois chrétiennes et non chrétiennes ; il y a des Phèdres japonaises ou africaines, toutes construites sur la mise en branle d'un référentiel puissant. De ce fait, il apparaît que le mythe de Phèdre peut s'inscrire dans un cadre interculturel car il devient un schème universel dans toutes les cultures.

Mais les œuvres contemporaines bien que radicalement opposées par leur écriture et leur esthétique, ont en commun outre la présence mythique, un nouveau rapport au monde. Elles 
s'attachent à dénoncer son impossible représentation et à déconstruire point par point les a priori qui le constituent. Plus que jamais, c'est parfois la décontextualisation de l'ancien mythe qui intéresse aussi dans ce qu'il a de transgressif, de contraignant et de novateur pour la littérature dramatique. Pour autant, choisir le mythe de Phèdre pour représenter un monde désabusé, tourmenté et sans repères n'est pas sans conséquences. A travers toutes les Phèdres produites et reproduites, touchant au sensible, remuant des sensations, nous cherchons à dénouer, à révéler notre rapport au désir, à la violence, la douleur, la culpabilité, l'humour pour, peut-être, mieux les appréhender ou nous réconcilier un peu plus avec nos corps, nos sentiments enfouis, la nature et les forces invisibles qui la mènent. Passant de la passivité à l'activité et vice versa, d'espaces clos à d'autres plus ouverts, nous touchons là où le plaisir et la peine, semblent être les deux faces de la même médaille.

Le choix de Yannis Ritsos, de Sarah Kane, de DidierGeorges Gabily et de Ximena Escalante que nous allons présenter dans la suite peut apparaître comme surprenant dans la mesure où ces auteurs largement reconnus ont habitué le public à des œuvres où la matière est tout aussi novatrice que l'écriture. Or, les œuvres choisies, sont des œuvres couronnées de succès comme en témoignent les nombreuses rééditions. En plus, nous avons choisi ces auteurs et leurs œuvres, parce qu'ils sont tous issus de cultures différentes quoiqu'ils traitent d'un même personnage, Phèdre, transposé dans une époque contemporaine.

Si cette nouvelle parole trouve ses origines dans le passé mythique de Phèdre et d'Hippolyte, elle est maintenant prétexte aux interrogations angoissées sur un monde contemporain violent et noir, afin que le théâtre, de nouveau miroir du monde, puisse faire transparaitre, dans cette tentative de représentation, une note d'espoir. Qu'elles soient antiques, classiques ou contemporaines, toutes les Phèdres parlent parce qu'un trop-plein 
de douleur les étouffe. Chez Racine, par exemple, Phèdre tombe malade de ne pas oser formuler son désir. La tragédie avance par aveux successifs. Chez Ritsos, l'aveu survient après une longue rétention au cours de laquelle se sont accumulés fantasmes et frustrations.

Phèdre de Yannis Ritsos (1909-1990), auteur emblématique de la littérature grecque du XXe siècle, est souvent mise sur les scènes du monde. L'œuvre a été écrite à la fin de la dictature des colonels en Grèce entre avril de 1974 et juillet 1975. C'est le dernier texte du recueil la Quatrième Dimension ${ }^{22}$, qui regroupe des textes concis ayant souvent l'allure de courtes mises en scène et qui sont inspirés par la mythologie antique. On a vu qu'ils relevaient d'une même préoccupation : celle du sujet, en tant que corps ayant une histoire, en tant que volonté affirmant son autonomie, en tant qu'être social soucieux d'une rencontre vraie. D'ailleurs, Ritsos ne fut pas le seul auteur grec à se servir de la méthode mythique, considérée comme l'utilisation du mythe à toutes sortes de fins ${ }^{23}$. Mais Ritsos se tourne vers le mythe selon un cheminement spirituel qui lui est propre et qui peut éclairer les raisons qui ont présidé au choix de son thème. De même, les enjeux de l'intertextualité mythologique semblent convergents chez lui. Il a écrit plusieurs monologues théâtraux dans lesquels

${ }^{22}$ Cycle réuni en grec sous le titre de Quatrième dimension et qui comprend seize monologues écrits entre 1948 et 1972, dont La maison morte(1960), Philoctète (19631965), Ajax (1967-1969), Perséphone (1965-1970), Chrysotémis (1967-1970), Hélène (1970), Ismène(1966-1971) et Phèdre (1974-1975).

\footnotetext{
${ }^{23}$ Michalis Pieris signale en se référant à l'utilisation fréquente du mythe chez Ritsos et les autres poètes grecs: "I'objectif de la caméra poétique se focalise sur le passé mythique utilisé comme ligne de mire pour viser le présent historique, en règle générale à

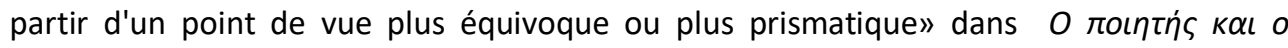

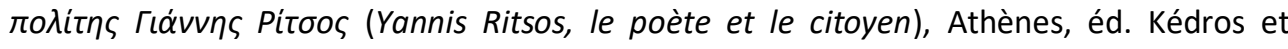
Musée Bénaki, 2008.
} 
s'expriment des personnages issus de la tragédie antique. En superposant les époques et en faisant des incursions dans la Grèce du XXe siècle, il amène ses personnages à une méditation distanciée sur le sens d'une vie et d'une action, la fuite du temps, la vieillesse. En liant les racines d'une tradition plusieurs fois millénaire au monde d'aujourd'hui, en passant par la Grèce des colonels, Ritsos obtient le résultat surprenant de créer des personnages auxquels nous puissions nous identifier, faits de sensualité, de perceptions en même temps que de réflexions sur les grands schémas de notre civilisation. Le monologue dramatique Phèdre invite à réfléchir celui qui ne se contente pas d'y voir une variation sur un thème déjà traité auparavant par Ritsos, c'est à dire de la dame mure qui s'adresse à un jeune homme. Cette Phèdre alors n'a plus d'autre choix que de laisser sa parole se déverser. Elle révèle les erreurs tragiques qui l'ont conduite à la catastrophe. Ritsos explore les contradictions de sa vie et en restitue le mystère par la magie de sa langue poétique. Comme une vague lente, les sons forment des mots qui prennent sens peu à peu avant de se déliter. Phèdre est folle de douleur, comme toutes les autres Phèdre qui vivent leur dernier jour et savent que leur aveu précède leur mort. Elle ose dire l'indicible. Elle incarne la part sensuelle existant en chacun d'entre nous. Son récit parle de sexe, de sang et de choses très concrètes. Sa parole sensuelle et flamboyante dégage une forme de sensorialité rayonnante, qui donne une résonance moderne à la déclaration d'amour, à son affirmation renouvelée de l'éternité du désir. Phèdre de Ritsos est une figure humaine qui est saisie dans un moment crucial de son existence, parvenue à un point extrême où ne semble plus s'ouvrir aucune issue. Paradoxalement le mal-être du personnage est associé à un exaltant sentiment de liberté, né, précisément, de la prise de conscience de sa condition humaine sur terre... La langue poétique se construit autour d'une problématique et d'un projet esthétique fédérateurs : le mystère 
secret de l'existence y est conçu comme un véritable processus épiphanique de déclenchement d'une prise de conscience nécessaire du désir de se libérer, au pire, de dénoncer, au mieux, de faire changer un état de fait. Pour autant, il n'y a ni palais, ni chevaux, ni sang versé. Seulement le monologue d'une femme, seule dans sa cuisine, une tasse de café à la main. Le quotidien dans toute sa platitude. Un monde sans surprise. Pour nous rappeler, comme l' a déjà signalé la critique littéraire, que pour Ritsos l'Histoire s'inscrit sur les choses insignifiantes ${ }^{24}$. De la tragédie, Ritsos ne garde que le cœur : le ressassement d'un désir fou - vécu à la fois comme une libération et un enfermement. Loin de plonger le sujet dans une forme d'obédience collective et passive, le recours au récit et l'allusion permet à l'être d'exprimer sa liberté intérieure. Une liberté intérieure qui n'est qu'une peau de chagrin si ne le remplit pas le poids du dehors:

... L'extérieur nous pénètre - une acceptation générale comme
le destin on se remplit soudain jusqu'à étouffer; on prend conscience du vide précédent ; ce vide n'est plus tolérable (et où trouver la plénitude? On étouffe). La sainteté du dénuement -

c'est ce que tu disais je ne me souviens plus bien : (que disais-tu au juste: du dénuement ou du refus?); des paroles vraiment insensées $^{25}$

Phèdre évoque le froid délire du désir et son trouble, dans le flot de ses souvenirs d'enfance, de ses rêves, de ses cauchemars. Un fleuve d'images qui déferle, aux portes de la folie, de l'errance et

\footnotetext{
${ }^{24}$ Comme le note Marangopoulos, "I'histoire non écrite de l'ordinaire » dans $O$ roıntís

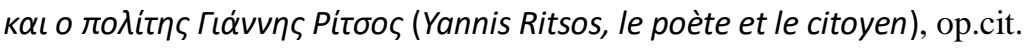

${ }^{25}$ Phèdre, dans Chrysotémis, Phèdre suivi de Le Sondeur et de Le Heurtoir, traduit par Gérard Pierrat, Gallimard, 1980.
} 
de la détresse, une recherche désespérée d'un hypothétique devenir. Hippolyte écoute et son silence impose à Phèdre de choisir, d'aller au bout d'elle-même. La réponse d'Hyppolite n'est pas dans les mots. Phèdre le condamne, et se condamne elle-même, choisit leur mort comme seule alternative. N'oublions pas encore que les personnages dans la poésie de Ritsos sont souvent allégoriques qu' il faut chercher leur filiation au-delà des apparences immédiates car parfois le poète nuit au penseur. Sans oublier que Yannis Ritsos situe la rencontre de Phèdre et d'Hippolyte dans la Grèce contemporaine. Chez lui, le contenu du mythe de Phèdre s'est élargi à des considérations plus philosophiques, relevant moins, d'un engagement immédiat, d'une prise de position dans l'actualité. On voit bien que l'expérience de la perte de soi décrite dans Phèdre, dépasse le cadre des œuvres plus «politiques» de Ritsos, celles où il est question notamment des traumatismes de l'histoire grecque récente. Nous y trouvons à la recherche des morceaux de Phèdre et d'Hippolyte comme autant de lambeaux de vérité à récupérer, leur amputation physique promeut la douleur et donc le savoir. Au total, ce qui ressort de la Phèdre de Ritsos est qu'une insertion aussi précise de l'action dramatique dans le mythe, qui constitue l'écriture dramatique en un espace de réinterprétation des textes et de la mémoire collective culturelle, apparait aujourd'hui presque exceptionnelle.

Bien autrement en va-t-il de la trilogie Gibiers du temps (1994-1995), de Didier-Georges Gabily (né en 1955) ${ }^{26}$, fractionnée en trois «époques »: Thésée, Voix, Phèdre, fragments d'agonie. Une trilogie née selon l'auteur de son désir

\footnotetext{
${ }^{26}$ Les pièces de Gabily sont comme des prolongements de mythes. Les héros mythiques des fables anciennes ne peuvent mourir et continuent à hanter le présent, malgré leur agonie au sens littéral : Don Juan dans Chimère et autres Bestioles, Phèdre et Thésée dans Gibiers du Temps.
} 
de créer «sa » Phèdre pour montrer : «Le monde d'aujourd'hui visité au travers de ce qui dure de ce mythe, de ses héros (fatigués ou pas), des passions qu'il véhicule ${ }^{27}$. Sur les planches apparaissent des figures incertaines de leur identité et troublées par ce qu'elles font. Toute l'histoire évoque le destin d'une Phèdre sans âge, dévoreuse d'hommes : chaque année, le jour anniversaire de la mort d'Hippolyte, elle se fait livrer un amant qui sera mis à mort après l'avoir fécondée ; Acamas et Démophon, les deux fils qu'elle a eus de Thésée, sont chargés de cette chasse. Rencontrant sur leur chemin leur père, revenu des Enfers et qu'ils ne reconnaissent pas, ils le mènent à Phèdre en un long parcours rythmé de meurtres, d'incestes, d'humiliations. Quand Thésée reconnaît Phèdre il lui dit : «Ne me dis pas qu'ici ce n'est pas l'enfer, toi aussi. C'est l'enfer. Encore. Bien que tes mains soient douces. Ne me dis pas que je suis revenu chez moi. Ce n'est pas chez moi du tout ». Avec la fin de Thésée, c'est tout le mythe qui s'affole et se trouve comme pris à l'envers. Gibiers du temps fait tout le contraire de ce que l'histoire nous livre. Au lieu d'être sauvé des enfers par Héraclès, Thésée lui doit maintenant la mort. Cypris-Aphrodite elle aussi se retourne contre l'origine : elle qui avait organisé la destitution d'Hippolyte pour se venger de l'affront de ce jeune-homme qui ne manifestait aucun intérêt pour l'amour des femmes, maintenant décide de se venger de celui qui incarne la virilité amoureuse : elle prépare la mort de Thésée et de Phèdre, les seuls qui assurent la suite d'Amour, la force de propagation de la filiation. Là aussi toute la pièce se construit sur la mise en branle d'un référentiel puissant du mythe ancien.

En résonance avec l'écriture transgénérique du théâtre contemporain paraît aussi la dramaturge anglaise Sarah Kane (1971-1999). Elle s'est donnée la mort, après avoir livré au

${ }^{27}$ Gabily Didier-Georges, Gibiers du temps. 
patrimoine théâtral cinq pièces (Anéantis, L'Amour de Phèdre, Manque, Purifiés et 4.48 Psychose) qui balancent entre hyperréalisme et poésie onirique et qui sont considérées comme des apports majeurs à la littérature dramatique. «L'Amour de Phèdre » (1996) relève du In-Yer-Face Theatre ${ }^{28}$ («Thêatre coup de poing » qui regroupe des dramaturges anglais des années 1990). Graham Saunders parle de «new Brutalists » pour désigner des auteurs comme Sarah Kane dans «l'Amour de Phèdre $»^{29}$. On y retrouve une théâtralité explosive, une poésie des ruines avec un fort impact émotif, un humour noir, de la perversion voire du sadisme. La chair est à vif, sans protection ; la violence, la souffrance qui mettent en place, une dynamique d'insatisfactions en chaîne sont nues ; finalement, la mort est inéluctable. Influencée par Edward Bond et Howard Barker, elle est une dramaturge politique ${ }^{30}$ qui traduit le politique en termes intimes. Elle parle du général par le biais de l'expérience individuelle de Phèdre et d'Hippolyte ; du macrocosme à travers le microcosme de ces deux héros. Kane fait référence explicitement à la famille royale anglaise. Sa pièce est un cri de désespoir dont l'écriture fonctionne tout d'abord par des chocs visuels, pour évoluer vers une esthétique sonore et vocale. Comme toutes les pièces de Sarah Kane Phèdre est exigeante et impose au spectateur un choix de nature éthique qui ne peut laisser indifférent. Il s'agit d'un théâtre de l'expérience ultime où se posent les questions de la capacité du langage à dire, dans ce monde qui en a fini avec l'humanisme. Kane transpose les personnages dans le monde contemporain sans marquer en rien notre distance à leurs sources : Phèdre et Hippolyte n'ont chez

\footnotetext{
${ }^{28}$ Selon l'expression d'Aleks Sierz qui a publié un livre pionnier: In-Yer-Face Theatre. London, Faber, 2001.

29 Voir Graham Saunders, Love or Kill Me. Sarah Kane and the Theatre of Extremes. Manchester, Manchester UP, 2002.

30 Voir l'entretien de Sarah Kane avec Nils Tabert, "Une conversation de Sarah Kane avec Nils Tabert", Outre Scène, n¹, Théâtre national de Strasbourg, février 2003, p. 68.
} 
eux que l'âge de leurs rôles, non celui des textes antérieurs par lesquels nous les connaissons. L'Hippolyte de Kane, adolescent, régressif et obsédé sexuel, meurt éventré par Thésée après avoir repoussé Phèdre dont l'amour, il le dit, ne ferait que le plonger plus profondément dans sa dépression. Ici, Hippolyte réduit son existence à la consommation (de junk food, de jouets, de gadgets technologiques, etc.) et à la contemplation passive (la télévision est allumée durant deux scènes sur les trois qu'il passe au palais). Il agit comme un verre grossissant qui fait voir, par l'excès de sa figure, la corruption à l'œuvre dans la famille royale, lieu de pouvoir et de richesse. Il dit notamment à Phèdre : «I was born into this shit, you married it $\gg$ (p. 76). Le travail de Sarah Kane sur le mythe de Phèdre consiste à débrider la tragédie de ses règles de pudeur, de retenue et d'immobilisme. Le sexe devient indissociable de l'amour et se retrouve au cœur des discussions. Le sang ne coule plus en coulisse et la mort n'est plus monologuée : ils sont offerts sur un plateau. L'œuvre de Sarah Kane est le négatif de la photographie de Racine. Ce postulat nourrit notamment la volonté de dépasser le bouclier charnel recouvrant le cœur de poésie omniprésente chez cette dramaturge. On peut d'ailleurs remarquer chez Sarah Kane que par ses références bibliques, l'atmosphère scénique s'éloigne de l'antiquité grecque et son polythéisme au profit de tableaux chrétiens.

La fable mythique, dans les textes de Gabily et de Kane, n'est plus que sa propre grimace, la matrice d'une traversée de la souffrance où ne se jouent ni la liberté ni la dignité de l'homme face à son destin, mais les étapes grotesques et pathétiques d'un nouveau chemin de choix. Si les œuvres de ces auteurs font écho au mythe ancien de Phèdre et en font surgir les topoi, elles se donnent comme un théâtre "post-tragique », qui en explorant des formes ultimes fictives et théâtrales, délimite les limites d'une nouvelle tragédie, nihiliste le plus souvent (on n'y décèle 
pas catharsis, ni retour à l'ordre), en tout cas affranchie de la définition aristotélicienne.

C'est un cas différent que représente Ximena Escalante qui est née au Mexique en 1964. A la fois auteure reconnue, metteur en scène et journaliste, elle a étudié dans son pays d'origine de même qu'en Espagne. Elle revisite de grands mythes, comme celui de Phèdre, qui lui permettent d'aborder les thèmes de l'amour, de la passion non partagée, des conflits qu'ils génèrent et des enjeux de pouvoir. Sa pièce Phèdre et les autres grecques a été créée au théâtre El Granero de Mexico en 2002 et a pour thème l'accession d'une jeune femme mexicaine à la maturité sexuelle. . Cette relecture moderne du mythe de Phèdre explore les chapitres moins connus de la vie de celle-ci en utilisant l'histoire de Phèdre et d'Ariane tout en permettant une incursion mordante et unique dans l'imaginaire féminin. Ici, le sort d'Ariane, la sœur aînée, est tout aussi crucial que celui de Phèdre. Ce bref résumé ne rend malheureusement pas ce que le récit a de vivant. Il ne donne pas davantage la mesure de l'habileté exceptionnelle de l'adaptation qui réussit à dessiner la difficulté de savoir aimer ce qu'on aime, de dépasser les fatalités qui pèsent encore sur nous : le corps et ses limites, les désirs et les frustrations, la tendresse sororale et ses trahisons. "L'histoire d'Ariane et de Phèdre n'est qu'un prétexte pour évoquer la femme d'aujourd'hui, prise entre le désir de maîtriser sa vie ou ses amours et la difficulté de vivre ce désir jusqu'au bout et sans compromission» précise Patrice Pavis dans la préface de l'œuvre traduite en français ${ }^{31}$.

A côté de ces quatre auteurs nous pourrions aussi ajouter l' Hongrois István Tasnádi (1970-) mais aussi la Russe Marina Tsvetaïeva (1892-1941), qui signent des réécritures sur Phèdre particulièrement stimulantes. Chez Tasnádi, né à Budapest en

\footnotetext{
${ }^{31}$ Escalante Ximena, Phèdre et autres grecques, p.11.
} 
1970, Athènes représente le centre de la culture européenne et Thésée y figure le fondateur de la démocratie. Tasnâdi décompose la dramaturgie antique en faisant révéler en alexandrins les secrets de l'âme humaine par une chorale de ménagères. De même, sa Phèdre reste profondément d'Europe centrale. Marina Tsvetaïeva , attirée plutôt par Euripide, avait publié dans un magnifique langage poétique en 1928 Phèdre, le deuxième volet $d$ ' une trilogie inachevée qui raconte les amours de Thésée. Elle choisit de se centrer surtout sur la relation de Phèdre et de la Nourrice en la représentant comme une forte relation entre une mère et sa fille. Dans cette œuvre la Nourrice fait tout le possible pour détacher Phèdre de son vieux mari. De l'autre côté, Hippolyte est considéré comme l'Autre inaccessible, inconnu et inconnaissable, l'objet de désir qui conduit Phèdre à la mort. Hippolyte apparaît comme un jeune homme souffrant de sa mère décédée qui réfléchit sur son destin sans descendance, destin de celui qui se consacre à la déesse vierge Artemis. C'est en 1922 que Marina Tsvetaïeva part à l'étranger, afin de rejoindre son mari. Ils vivent d'abord à Berlin, puis à Prague, avant de s'installer à Paris. Phèdre, son sujet de prédilection devient alors la douleur de la femme apatride. Evacuée avec son fils dans le village tartare d' Elabuga, elle s'y suicide en 1941 comme Phèdre de Sénèque par pendaison peu après l'invasion allemande.

Nous continuons à y apercevoir des écritures avec le désir de mettre en résonance les formes antiques et modernes ; la passion, l'amour, le sexe, la mort : à travers le mythe de Phèdre on s'interroge sur la perte des utopies et des illusions, l'emprise de la passion sur la raison, la naissance du sentiment de culpabilité et la prise de conscience du péché, le rétrécissement des valeurs sociétales et sa représentativité théâtrale. Si bien qu'en dernière analyse on peut dire qu'il existe dans toutes les Phèdres partout au monde un univers qui englobe tous les autres, 
un univers où tous les éléments, voire ceux en apparence les plus étrangers au mythe, acquièrent droit de cité.

Selon Giorgio Agamben, le tragique de notre époque tient au fait que l'homme est abandonné au langage, "sans avoir une voix ou une parole divine qui lui assure une issue hors du jeu des propositions signifiantes ». Les réécritures de Phèdre sont ainsi partie intégrante d'un mouvement de rénovation et elles puisent dans le mythe et dans le passé, elles font aussi retracer la situation humaine au cœur du monde moderne, prémices des écritures tout au long du XXe et du début du XXI siècles ${ }^{32}$. Cette renaissance de l'écriture vient dès lors inscrire le mythe ancien dans le temps présent, si le noyau demeure, il n'est plus dit de la même façon, ni entendu de la même façon. Ainsi, Claudia Bosse envisage-t-elle le mot en l'air, hors du corps, comme une catastrophe, un déchirement et se propose d'interpréter Phèdre à la lumière de la théorie de « l'acte de parole », ou performativité du langage ${ }^{33}$.

Phèdre inspire ainsi l'envie d'explorer les systèmes de réception de l'objet théâtral et de se demander sur la manière dont son corps devient le lieu où se négocient les discours

\footnotetext{
${ }^{32}$ Dans ce sens, on a pu dire que Phèdre est " un mythe qui définit les postulats de la sensibilité littéraire de notre temps, en une époque dont la caractéristique essentielle est de sentir et d'imaginer l'homme comme placé dans un monde qui ne semble pas fait pour lui, d'où [...] l'effort éthique pour se sauver par soi-même » dans R.-M. Albérès La révolte des écrivains d'aujourd'hui, p. 15.

${ }^{33}$ Le postulat de cette théorie, initiée par John L. Austin dans son livre Quand dire, c'est faire (1962), suppose

qu'un énoncé n'est pas seulement constatatif mais peut modifier l'ordre du monde. II en arrive à la conclusion que tout énoncé est performatif, même les énoncés tenus pour constatatifs : parler c'est toujours agir. Sa recherche sera poursuivie par John R. Searle, dans Les actes de langage (1972), puis, plus récemment, par Judith Butler, dans son ouvrage Le pouvoir des mots, politique du performatif (2004). En se basant sur les discours de haine (hate speech) homophobes, racistes ou sexistes, sur la pornographie et la censure, elle pose, dans la lignée de Foucault, les grandes lignes d'une théorie de la puissance d'agir linguistique (linguistic agency), d'une politique de la re-signification, et critique les tentatives de police des discours.
} 
politiques et moraux actuels. Décrivant son expérience intérieure, elle décrit également, avec une grande clairvoyance, notre ère et c'est ce qui donne tant de pertinence à sa réapparition continuelle. De même elle nous rappelle qu'Il y a identité profonde entre la scène intime et la scène publique. En ce sens les réecritures de Phèdre œuvrent pour une nouvelle fondation qui rapproche tous les auteurs qui pour ce faire, éprouvent le besoin de mette en parallèle cette fondation et celle qui a eu lieu des siècles et des siècles auparavant lorsque les Grecs anciens ont eux aussi senti la nécessité de mettre en scène les changements qui se jouaient dans leur cité.

Dans toutes les œuvres examinées ici, la parole de Phèdre tapie à l'ombre du corps, dans les tréfonds de l'âme, est ramenée à la lumière dans l'acte de dire et dans le geste. Alors seulement

elle devient visible, perceptible et communicable. Cela permet aussi d'ouvrir une réflexion contemporaine sur la politique des rapports humains et des rapports amoureux: la mise sous contrôle du sujet et de ses passions devient affaire d'Etat; irrationnelles et capricieuses, celles-ci menacent la raison d'Etat. C'est pourquoi, Phèdre ne cesse jamais de se présenter comme une figure transgressive, qui fascine en même temps. Si la forme, les contours du mythe de Phèdre changent à travers ses variations dans les siècles, passant du lyrisme et de la poésie au dialogue elliptique et au langage rigide, le noyau du mythe reste toujours le même. Il n'est qu'une mise au point qui cerne les éléments traditionnels dans le mouvement nouveau. Que nous attribuions les causes du drame à la toute puissance des dieux, à la victoire de la volonté humaine, à l'affirmation de la puissance individuelle, ou à la tension, au combat entre la logique et le divin, le contrôle et l'incontrôlable.

Un constat inévitable porte sur le rôle radicalement changé de la femme dans le mythe. Depuis les tragédies 
d'Euripide, de Sénèque et de Racine jusqu'à ses réécritures aux XXe et XXI siècles, on verra l'éclairage du récit se déplacer du héros masculin, Hippolyte, vers le personnage féminin ; le tragique être mis en cause ; la conscience de l'inceste hésiter entre la relation de la marâtre au fils de son époux, et celle des deux sœurs (Ariane et Phèdre) au même homme, Thésée. Un renversement, un revirement semble se produire par lequel Phèdre acquiert une position beaucoup plus active «exigence fondamentale de notre temps $»^{34}$, beaucoup moins dépendante que ce que nous propose le mythe traditionnel. En somme, Phèdre apparaît en héroïne de l'action directe. Selon les théoriciennes américaines du mouvement «revisionist mythology» ${ }^{35}$, il s'agirait là d'un processus typique et nécessaire, marquant un étape importante dans l'histoire de notre civilisation, avec le rejet des valeurs patriarcales et la poussée d'une conscience davantage matrilinéaire sinon matriarcale . Ceci ne suffit peut-être pas à expliquer les changements advenus dans les arts - et surtout le fait que pour la majorité des artistes contemporains, ce soit encore la figure de Phèdre qui semble se manifester sans hésitation, qui demande à surgir, s'exprimer par sa propre parole et qui a pris conscience du pouvoir verbal sans renoncer au modèle antique. Ainsi, cette Phèdre propose un langage «post-linguistique ${ }^{36}$ qui a à nouveau confiance en lui.

Admettons que si les œuvres citées dans notre parcours sont apparues plus ou moins proches les unes des autres grâce à la figure de Phèdre revisitée, les confronter a aussi permis de mette en évidence la réalité des écritures uniques qui, si elles s'interrogent toutes sur leur temps national (par exemple, en

\footnotetext{
${ }^{34}$ CF. R.-M. Albérès La révolte des écrivains d'aujourd'hui, p. 12.

${ }^{35}$ dont témoignent entre autres les écrits de Barbara Walker

36 Dans ce que Steiner nomme le post-linguistique : " phase de dépérissement du langage, de formes postlinguistiques et peut-être d'un silence, au moins partiel ? " dans Steiner Georges, (tr. Celli, Rose), La Mort de la tragédie, Paris, Gallimard, 2002.
} 
Grèce, en Angleterre, en France, au Mexique, en Europe de l'Est ou en Russie) et sur le chaos mondial, ne procèdent pas de la même façon et n'en tirent pas des conclusions identiques. Mais ces œuvres dont on a pu voir ici un aspect, ne cessent de retravailler la matière ancienne sur Phèdre et Hippolyte et d'y trouver un sens fondamental dans ce ressassement.

D'Homère au foisonnement de visages discordants que les siècles lui donnent en leurs œuvres littéraires autant qu'en leurs mises en scène, Phèdre semble bien avoir joué le rôle d'incitatrice d'un mythe littéraire, défini en l'occurrence comme un éternel retour infiniment changeant. Aujourd'hui, il ne fait plus de doute qu'une certaine unicité et une cohérence entre les

œuvres dramatiques et notre société, au sein de laquelle les Phèdres s'enracinent et permettent à la dramaturgie de nos jours de se poser comme étant existante et depuis, foisonnante. En dernière analyse, le mythe classique s'enrichit de variantes dynamiques novatrices qui mettent en valeur leur fonction symbolique. Figure ambivalente, Phèdre apparaît dans la diachronie souvent sous des aspects méconnus de son mythe et émerge dans l'imaginaire littéraire en apportant son héritage ancien. A cet égard, le mythe de Phèdre constitue un authentique archétype devant lequel les auteurs postérieurs doivent, consciemment ou non, abdiquer une part de leur originalité. Phèdre, c'est certes, la conscience tentant perpétuellement de concilier la lucidité et l'acceptation de soi dans la lumière de la lucidité. Mais c'est aussi certaine impossibilité de «bonheur normal » hors de tout compromis. Tout compte fait, Phèdre, figure atemporelle et complexe, continue à échapper toujours à toute notion de moralité et illustre la bienveillance et la monstruosité à la fois d'une féminité sacrée éternelle, ancrée dans le fonds culturel prégnant notamment greco-romain. 


\section{Bibliographie}

Agamben Giorgio, La communauté à venir. Théorie de la singularité quelconque, Paris, Seuil, coll. «La librairie du XXIe siècle », 2004.

Albérès Réné-Maril , La révolte des écrivains d'aujourd'hui, Paris, Corrêa , 1949.

Barrault Jean-Louis. Phèdre, Seuil, « Mises en scène », 1946.

Barrett William Spencer, Euripides Hippolytos, Edited with Introduction and Commentary, Oxford University Press, 1964, 1992.

Barthes Roland, Sur Racine, Seuil, 1963.

Battesti Jean-Pierre, Chauvet Jean-Charles et Biet Christian (dir.), Tout Racine. Dictionnaire : l'homme, l'oeuvre, la postérité, Paris, Larousse, 1999.

Brunel Pierre (sous la dir. de), Dictionnaire des mythes littéraires, Du Rocher, 1988.

Brunel Pierre, Mythocritque, théorie et parcours, Paris, PUF, 1992.

Carpenter Thomas et Robert Gula, Mythology Greek and Roman. Wellesley Hills, Massachusetts: The Independant School Press, 1977.

Demon Paul et Lebeau Anne, Introduction au théâtre grec antique, Paris, Le livre de poche, 1996.

Diel Paul, Le symbolisme dans la mythologie grecque. Paris, Payot, 1952. 
Dupont Florence, Le théâtre latin, Paris, Armand Colin, 1988.

Durand Gilbert, Structures anthropologiques de l'imaginaire. Paris, Bordas/Dunod, 1969.

Durand Gilbert, Figures mythiques et visage de l'œuvre, de la mythocritique à la mythacritique, Paris, Berg, 1970.

Eliade Mircea, Aspects du mythe. Paris: Gallimard, 1963.

Escalante Ximena, Phèdre et autres grecques, (traduit de l'espagnol), La Guillotine, 2004.

Gabily Didier-Georges, Gibiers du temps, Paris, Actes Sud, 1995.

Gliksohn Jean-Michel, "Le mythe de la guerre des sexes », dans Mythes, images et représentations, actes du XIVème congrès- Limoges, 1977- de la société Française de Littérature Générale et Comparée, sous la direction de J. M. Grassin, Limoges, trames et Paris, Didier, 1981.

Foley Helene, Female acts in Greek Tragedy, Princeton University, 2001.

Hassan Ihab. The Dismemberment of Orpheus. Toward a Postmodern Literature. New York: Oxford University Press, 1971.

Jouan Francis. "Le retour aux mythes grecs dans le théâtre français contemporain », Bulletin de l'association Guillaume Budé, Juin 1952. pp. 62-80. 
Kane Sarah. Phaedra's Love, 1996 ; L'Amour de Phèdre, traduction française de Séverine Maglois, Paris, L'Arche, 1996.

Kristeva Julia, Pouvoirs de l'horreur: essai sur l'abjection. Paris: Seuil, 1980.

Langridge Natasha, Stephenson Heidi, Rage and reason. Women playwrights on playwriting. London, Methuen, 1997.

Larrington Carolyne (sous la dir. de). The Feminist Companion to Mythology. London, Pandora Press, 1992.

Maulnier Thierry. Lecture de Phèdre, Gallimard, 1943.

Pageaux Daniel-Henri, La littérature générale et comparée, Paris, Armand Colin, 1994.

Prokopaki Chryssa, «The cycle of Yiannis Ritsos`mythological poems», www.greece.poetryinternational.org/cwolk/view/21906.

Regan Eichelburg, Racine's Definition of the Heroine and the use of Euripides, Columbia University, 2001.

Ritsos Yannis, Phèdre (1978), dans Chrysothémis ; Phèdre ; (suivi de) Le Sondeur ; Le Heurtoir, traduit du grec par Gérard Pierrat, Gallimard, 1980.

Romilly Jacqueline (de), La modernité d'Euripide, Paris, P.U.F., 1986.

Sénèque, Phèdre, dans Tragédies, Tome I, Paris, Les Belles Lettres, 1961. 
Schlegel August Wilhelm, Comparaison entre la Phèdre de Racine et celle d'Euripide, Oxford, A.L. Pollard, 1962.

Soare Antoine, «La rencontre de Phèdre et d'Hippolyte de Sénèque à Racine », in Etudes françaises, vol. 44, $\mathrm{n}^{\mathrm{0}} 2$, pp. 119- 135, 2008.

Tasnádi István, Phèdre 2005, éd. Théatrales, 2006.

Tschiedel Hans Jürgen, Phoedra und Hippolytus. Variationen eines tragischen Konfliktes, Erlangen, 1969.

Tsvetaïeva, Marina, Phèdre. Actes Sud, 1991.

Walker Barbara. 1983. The Woman's Encyclopedia of Myths and Secrets. San Francisco, Harper. 